Techniques \& Culture

\title{
Souffler pour ordonner
}

Une conque pour l'irrigation en Galice

\section{Fabienne Wateau}

\section{(2) OpenEdition}

Journals

Édition électronique

URL : https://journals.openedition.org/tc/2652

DOI : $10.4000 /$ tc. 2652

ISSN : 1952-420X

Éditeur

Éditions de l'EHESS

\section{Édition imprimée}

Date de publication : 12 décembre 2007

Pagination : 39-60

ISSN : 0248-6016

\section{Référence électronique}

Fabienne Wateau, «Souffler pour ordonner », Techniques \& Culture [En ligne], 48-49 | 2007, mis en ligne le 20 juin 2010, consulté le 29 septembre 2022. URL : http://journals.openedition.org/tc/2652 ; DOI : https://doi.org/10.4000/tc.2652 


\section{Fabienne Wateau*}

\section{Souffler pour ordonner Une conque pour l'irrigation en Galice}

Dans le sud de la Galice, une conque était autrefois utilisée pour sonner les tours d'eau dirrigation en été. Cette pratique ancestrale a cessé d'être opératoire dans les années I980 quand la cimentation de la rigole principale et létablissement d'un document officiel ont été réalisés. Quelque vingt ans plus tard, à l'occasion d'un essai de reconstitution filmographique de cette activité, le son de la conque a ravivé chez les villageois une mémoire empreinte de nostalgie. Lapproche ethnographique vise à montrer les logiques qui présidaient à lorganisation sociale de lirrigation en été et à souligner limportance de cet objet dans le paysage sonore et affectif de la vallée.

Eau, irrigation, Galice, conque, instrument d’appel, cognition, mesure, Espagne.

La conque espagnole présentée ici est un instrument d’appel utilisé en période estivale dirrigation. Son étude sinnscrit dans une série qui porte sur les instruments de mesure de l'espace, du temps et de l'eau, organisée en un double triptyque qui comprend trois objets et trois films. À chaque objet correspondent un film et un article. L'objet est alors étudié de façon individuelle et spécifique, dans sa relation à l'espace, à la mesure, à ses utilisateurs, à la technique et à ses représentations. Les trois objets ont pour particularité d’appartenir à la même aire géographique, à un espace situé de part et d'autre de la rivière transfrontalière Miño ou Minho, comprenant au nord le canton d'Arbo en Espagne et au sud le canton de Melgaço au Portugal. Il s'agit d'un paysage de moyenne montagne extrêmement arrosé par les pluies océaniques, regorgeant de sources et de cours d'eau, et doté en vallée et sur les flancs de coteaux de parcelles de minifundia en terrasses destinées, jusqu’aux années 1990, à la polyculture vivrière irriguée. Avec

"CNRS Nanterre (UMR 7186), Université Paris X. 
l'entrée de l'Espagne et du Portugal dans la communauté européenne en I986, la restructuration et la réorientation d'une grande partie des parcelles de minifundia vers une monoculture intensive de vigne, et plus particulièrement vers un cépage noble de vin vert appelé alvariño $(\mathrm{E})$ ou alvarinho $(\mathbf{P})$, aujourd'hui fort bien côté sur les marchés nationaux et internationaux, ont modifié le rapport à la terre et à l'eau. L'économie locale s'est adaptée rapidement à une production spécialisée et commercialisable, laquelle ne recourt plus systématiquement et avec la même intensité à l'arrosage des parcelles en été. Par-delà la géographie et le partage séculaire d’un même comportement économique, cet espace transfrontalier présente d'autres caractéristiques communes : un passé de dictature, une inscription paysanne forte avec un pourcentage encore élevé de petits propriétaires terriens, une langue parlée proche, un discours identitaire actuel sur les zones de périphérie souffrant d'isolement, une augmentation progressive du secteur tertiaire et du tourisme vert, des échanges nombreux, etc. Soit un ensemble d'éléments qui m'ont conduite à considérer l'espace comme culturellement homogène et donc comparable. Sur le terrain, les trois artefacts étudiés se situent à environ cinq kilomètres les uns des autres, servant tous au partage estival de l'eau d'irrigation. Pourtant, c'est leur différence de nature et ce que je considérais être une abondance des procédés techniques et logiques pour partager l'eau, dans un espace si restreint, et de surcroît pour une activité agricole connue et partagée de tous, qui ont attiré mon attention et invité à rapprocher les objets et les logiques de cette vallée dans une même série cognitive. En d'autres termes, dans ce contexte d'abondance en eau, c'est une autre forme d'abondance qui a retenu mon attention, ou encore une absence patente de modèle unique ou uniformisé. Ces instruments sont une canne de roseau graduée qui sert à partager des volumes d'eau retenus dans de petits bassins-réservoirs de flancs de coteaux, une pierre de partage ou sorte d'horloge solaire à partir de laquelle sont découpés des espaces-temps d'irrigation et une conque marine à embout sectionné qui, comme nous allons le voir, sert à signaler le roulement des bénéficiaires d'eau sur le parcellaire irrigué. Les trois films ont été réalisés ; deux des trois articles sont publiés ${ }^{1}$. La présentation de la conque est donc le troisième et dernier article de la double série, laquelle sera bientôt analysée en soi et conduira, dans une

\footnotetext{
1 Pour le détail de la présentation de ces instruments, voir les écrits Wateau (2001) pour la canne de roseau et Wateau (2006a) pour la pierre de partage ; pour les images voir les références filmographiques en fin d'article.
} 
publication ultérieure, à l'analyse comparée et croisée des objets, des pratiques et des schèmes de pensée. Écriture académique et écriture cinématographique seront alors mises en relation, de façon à en souligner l'apport mutuel et lintérêt de leur combinaison pour la recherche scientifique.

Pierre, conque et bâton, l'objet d'étude ne semble pas très sérieux. Pourtant, idéalement, il s'agit de montrer comment la lecture interprétative des objets peut ouvrir sur la lecture anthropologique des sociétés et plus particulièrement sur liidentité des sujets. La conque n'est pas l'instrument le plus simple à appréhender. Sa pratique tombée en désuétude depuis les années 1980 n'a pas permis ces longs séjours sur le terrain où l'observation des faits et gestes de chacun des utilisateurs comme du reste des membres de la communauté permet de documenter le carnet de terrain et de poser les bonnes questions, en situation. Cet article diffère des deux précédents par l'absence de données ethnographiques recueillies à partir d'observations directes. À Melgaço (P), où la pierre et la canne sont toujours utilisées, leurs habitués ont longuement été interrogés et suivis dans leurs pratiques comme dans leur sociabilité. À Arbo (E), la démarche a été différente. La conque à l'étude, qui appartenait au dernier sonneur du village d'Arbo, localement appelé Pépé (fig. 2), m’avait été donnée par lui il y a une dizaine d’année. Je cherchais alors côté espagnol d'éventuelles pierres de partage et cannes à mesurer l'eau pour mon étude comparée. Et jen avais trouvées. Les premières, bien qu'encore visibles, avaient complètement été abandonnées; les secondes, encore en usage mais tendant à disparaître, à la fois ressemblantes et assez différentes des cannes portugaises, mavaient fourni des pistes de réflexion fort utiles pour analyser les principes régissant les logiques de partage de chaque côté de la rivière ${ }^{2}$. Mon échange avec Pépé avait été bref. L'homme réservé et timide m’avait rapidement expliqué en quoi consistait son rôle de sonneur, puis il mavait cédé sa conque sans manifester d'attachement particulier à l'objet. Bien plus tard, en 2003, quand je suis retournée à Arbo avec lintention de réaliser un petit film reconstituant cette activité3, j’ai retrouvé Pépé mais aussi d’autres interlocuteurs, plus

\footnotetext{
2 Voir Wateau 2001.

3 Film réalisé aussi pour être présenté au colloque de la SIEF à Marseille en 2004, à la table ronde «Objets de rencontre, objets de disputes ou comment faire parler les objets des relations entre les sociétés de l'Europe et de la Méditerranée? », Wateau (2004).
} 
loquaces, dont d'anciens sonneurs disposés à jouer le jeu de la reconstitution et le président de l'association de la rigole d'Arbo. Plusieurs conques ont été sorties des placards, certaines plus grandes que d'autres, toutes ayant servi au partage de l'eau en période estivale d'irrigation. La conque était un objet connu de tous, un instrument familier attaché à une activité agricole précise, un élément du paysage sonore local. Chaque sonneur avait la sienne, qu’il avait gardée ou non au fil des années. Assez vite, cinq sonneurs et quatre conques avaient été retrouvés. Côté portugais, j’ai aussi cherché une conque et un sonneur de conque. Mais à Melgaço, le sonneur de conque pour lirrigation ne semble pas représenté. On trouve parfois chez les gens des conques à embout sectionné, lesquelles sont destinées, selon leurs dires, à signaler la présence des loups en montagne, à appeler un berger éloigné ou encore à décorer la maison. Mais l'existence d'un lien entre la conque et lirrigation ne m’a jamais été mentionnée : le lien a peut-être été oublié ou peut-être n'a-t-il jamais existé.

\section{Une conque, deux sonneurs et un cadastre}

La conque servait au partage de l'eau en période estivale d'irrigation sur le parcellaire irrigué d'Arbo. Celle qui est représentée ici est une petite conque terminale simple mesurant $\mathrm{I} 7 \mathrm{~cm}$ de long et pesant $\mathrm{I} 86 \mathrm{gr}$ (cf. fig I). L'orifice de la section qui forme l'embouchure est de $2 \mathrm{~cm}$ de diamètre, le tour complet large de la conque mètre $26 \mathrm{~cm}$ et le tour complet plus resserré $19 \mathrm{~cm}$; la profondeur est de $5 \mathrm{~cm}$. Son nom en latin est Charonia tritonis. Il sagit d'une conque à enroulement dextre, soit d'une conque ordinaire Localement, en galicien, elle est appelée corna ou corneta, ce qui signifie dans le dictionnaire usuel $^{4}$ : corne qu utilisaient les porchers pour appeler les cochons, ou encore, en castillan, cuerna : trompe de facture similaire à la corne de bœuf utilisée par les gardes et autres habitants de la campagne pour communiquer entre eux. Il s'agit d'un instrument d'appel, dont on peut imaginer que la substitution éventuelle par une corne bovine n'aurait guère modifié lintention et le résultat souhaité. Les conques utilisées à Arbo étaient généralement achetées au marché La Piedra du port de Vigo, qui se trouve à une soixantaine de kilomètres de là, dans des magasins de vente de

\footnotetext{
${ }^{4}$ Diccionario de la lengua española, Madrid, Real academia española, 1992.
} 
coquillages décoratifs ${ }^{5}$. Chaque sonneur achetait la sienne; il n'existait pas de transmission particulière d'utilisateur à utilisateur ou de génération en génération. Elles étaient ensuite coupées à la scie ou avec un autre objet tranchant. La conque est à la fois un objet banal, utile et esthétique, que l'on garde à la maison sur une étagère ou au fond d'un tiroir, en souvenir d'une activité.

L'usage semble ancien, remontant pour le moins à la fin du XIXe siècle, selon les archives de I843 qui attestent de la pratique. Pour les uns, la conque était encore utilisée en 1992, et avec plus grande certitude encore, en 1985; pour les autres, et notamment José Simón, ancien responsable de la confédération hydraulique, la conque a cessé d'être utilisée en I975, avec l'établissement du registre et du cadastre officiels des eaux de la rigole d'Arbo. Néanmoins, sur le document officiel de la délégation de Pontevedra, destiné à procéder à la cimentation de la rigole d'Arbo et datant de 1988 , lusage du sonneur est encore mentionné, reprenant les termes exacts du document de I843.
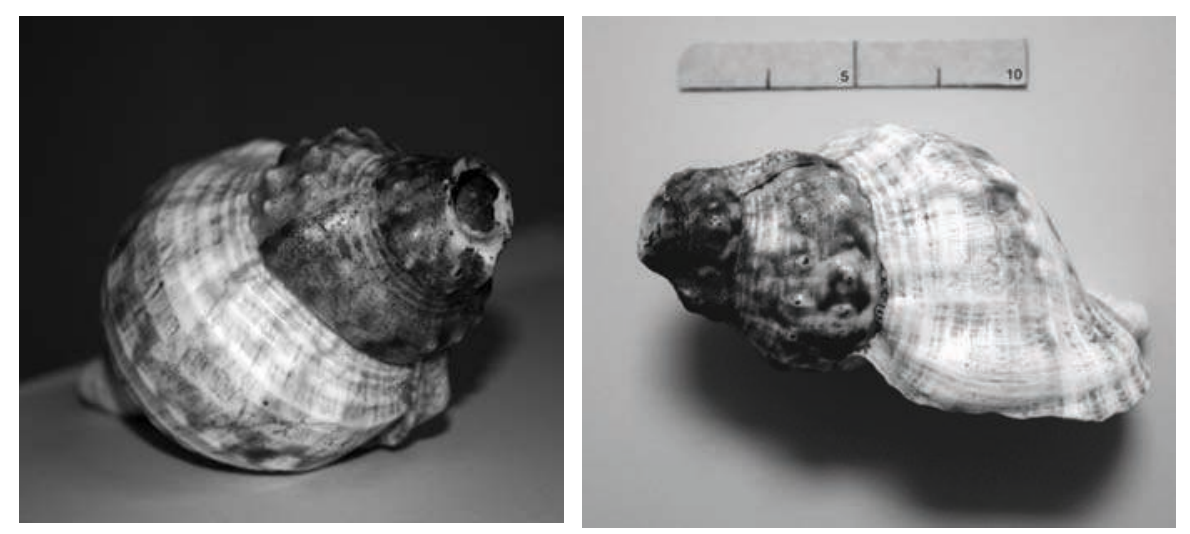

Figure 1. Conque pour sonner les tours d'eau à Arbo (cliché Frédéric Joulian).

Pépé, de son vrai nom José Gomez, exerça cette tâche de 1955 à 1975 ; il apparaît comme le dernier levador régulier de la rigole d'Arbo, assisté chaque année d'un autre sonneur qui, en alternance, parce que lirrigation se

5 Selon mes interlocuteurs de 2003. 
pratiquait de jour comme de nuit, le relayait dans son travail. Le titre de levador vient de levada, la rigole d’amenée, il signifie "responsable de la rigole " et par extension responsable de lirrigation, "conducteur" de l'eau. Son appellation ne dépend donc pas de son action de souffler dans la conque mais de sa responsabilité - et ce, même si extraire un son de la conque n'est pas forcément évident, comme on le voit dans le film (Wateau 2004). Chaque année, le dimanche précédant l'ouverture de lirrigation estivale, soit quelques jours avant le 26 juin, tous les propriétaires de parcelles qui irriguaient avec l'eau de la rigole d'Arbo se réunissaient pour désigner le responsable de l'été. On procédait alors à une enchère au moins disant, qui commençait par l'annonce d'une rémunération maximale fixée, à partir de laquelle les différents candidats enchérissaient à la baisse jusqu’à ce que celui qui propose finalement le salaire le moins élevé remporte la charge. Le gagnant des enchères devenait le responsable de l'été, jusqu'au II septembre, date officielle de fin dïrrigation estivale dans toute la vallée, pour la somme définie collectivement. Il était payé par chacun des irrigants au prorata de leurs avoirs en eau. Sa responsabilité consistait à conduire l'eau depuis la première prise jusqu'à la dernière parcelle, à l'extrémité de la rigole. Il lui incombait de souffler dans la conque les temps d'irrigation de chacun, c'est-àdire, à partir de la liste officielle des parcelles et des ayants droit quil avait en main, de sonner chaque changement de propriétaire: un coup sec pour marquer la fin d'un temps d'eau, un coup long pour signifier son début. Le découpage étant extrêmement méticuleux, la tâche n'était pas de tout repos. À chaque parcelle (au nombre total de 882 sur le cadastre de 1988), en effet, revenait un temps d'eau mesuré en minutes, et certains de ces temps se réduisaient parfois à des demi-minutes, selon la superficie du terrain. Une fois le temps d'eau imparti écoulé sur une parcelle, l'eau passait à la terre suivante dans le tour général. Elle était alors coupée par le bénéficiaire qui allait irriguer, lequel plaçait dans la rigole une vannette mobile appelée $t^{\prime} l^{6}$ pour orienter l'eau vers sa propre parcelle. Et ce, «dès qu’il recevait l'ordre du levador qui lui signifiait personnellement au moyen d'une cuerna (un coquillage marin) qu'il faisait sonner, $[. .$.$] il avisait aussi le temps aux futurs$ usagers " (Vázquez Martínez 1946 : 175). Dès quili prenait ses fonctions, tous les ayants droit devaient respecter le levadoret «se considérer sous ses

\footnotetext{
${ }^{6}$ Tol est le terme galicien qui renvoie à Tola en portugais. Le terme castillan est comporta.
} 
ordres, dans le cadre de ce qui avait été traditionnellement établi. Les deux levadores avaient une autorité pour élucider, résoudre, éclairer et devenir des témoins privilégiés en cas de conflits graves, quand la justice municipale devait intervenir, ou quand une résolution à l'amiable n'avait pu être trouvée pour régler le différend apparu au moment de la distribution de l'eau " (Vázquez Martínez I946 : 174). Souffler consistait done aussi à ordonner.

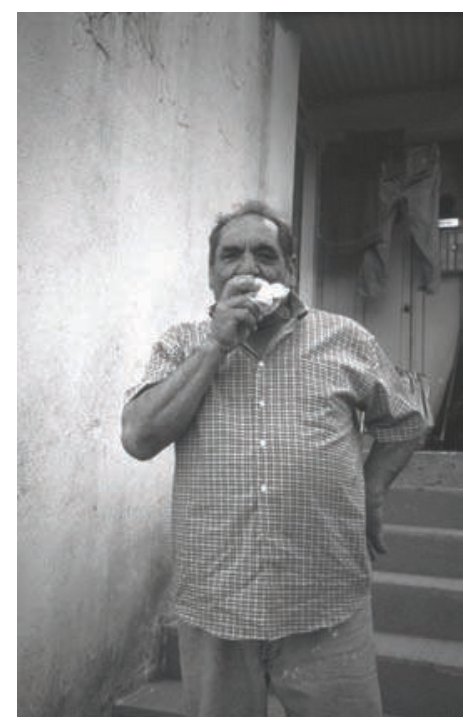

Figure 2. José Gomez, dit Pépé, dernier sonneur de conque à Arbo (cliché Fabienne Wateau).

Le parcellaire irrigué par la rigole d'Arbo, elle-même alimentée par des sources qui sourdent dans la partie moyenne des montagnes et par les eaux du Río Cea que l’on détourne à l'aide de prises d’eau, a entièrement été redessiné et posé sur le papier en I988 (fig. 3 ). Avant cette date, il n'existait pas de planche mais seulement des cadastres en forme de liste qui donnaient les temps d'eau de chacun. La superficie totale de l'aire irriguée couvre $546072 \mathrm{~m}^{2}$. Elle est distribuée en sept casales ou groupes qui comprennent chacun une série de parcelles. Ces parcelles sont situées le plus près possible de la rigole principale de distribution, à partir de laquelle bifurquent encore des canaux secondaires et des canaux tertiaires. Un tour complet dirrigation dure sept jours, correspondant aux sept casales, «de telle façon que lorsque 
commence le huitième jour, commence à nouveau le propriétaire le plus près de la première prise d'eau, comme si on se retrouvait au premier jour de lirrigation, et ainsi successivement" (Vázquez Martínez 1946: 175). L'eau s'écoule du premier vers le septième groupe de parcelles, à raison d'un groupe par jour, de haut en bas, par parcelles contiguës, les unes à la suite des autres. Cette distribution de l'eau est rationnelle d'un point de vue physique et économique. Elle respecte la gravité du terrain, faisant en sorte que l'écoulement se fasse le plus naturellement possible, du haut vers le bas. Elle a pour objectif d'optimiser les quantités d'eau acheminées, en évitant les déperditions liées aux trajets non nécessaires et en établissant un mouvement balayant sur les parcelles, lequel favorise limbibition des terres mitoyennes. Cette situation est fort différente de celle observée de l'autre côté de la rivière, dans la commune de Chaviães au Portugal, où, si le système est aussi gravitaire, la distribution de l'eau se fait selon une logique sociale et non pas strictement économique - nous aurons l'occasion d'y revenir plus loin.

À titre indicatif, en 1946 et d'après le cadastre de 1843 (appelé Casalmayor del año I843), si l’on en croit Vázquez Martínez, la distribution de l'eau sur les sept casales se répartissait en heures et en minutes de la façon

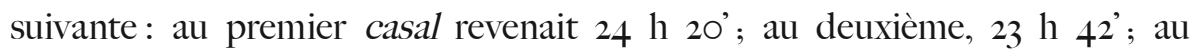
troisième, 23 h $5^{8^{\prime}}$; au quatrième, $24 \mathrm{~h} 22^{\prime}$; au cinquième $24 \mathrm{~h} 04^{\prime}$; au sixième, $23 \mathrm{~h} 32^{\prime}$ et au septième, 26 h I7'. Soit un total de I70 h $22^{\prime}$ par tour complet ${ }^{7}$. La plus grande part d'eau correspondait à $2 \mathrm{~h} 20^{\prime}$ et la plus petite à une demiminute. Sur le cadastre réactualisé de 1975 mais datant de 1952, il apparait que le premier casal comptabilise cette fois 1450,50 minutes $(24 \mathrm{~h} \mathrm{I}$ ) ; le deuxième $1397,5^{\circ}$ ( $23 \mathrm{~h} 29$ ); le troisième $\mathrm{I} 47 \mathrm{I}, 5^{\circ}$ ' $24 \mathrm{~h} 52$ ); le quatrième $1398^{\prime}$

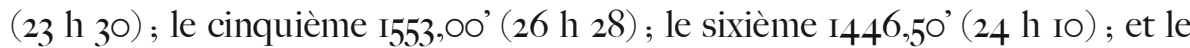
septième ${ }_{53} 6,00$ ' $(26 \mathrm{~h} 00$ ). La plus grande part d'eau correspond cette fois à 99 minutes $(2 \mathrm{~h} \circ 5)$ et la plus petite toujours à une demi-minute. Enfin, sur le cadastre de 1988, établi à l'occasion de la cimentation de la rigole et qui donne aussi les surfaces irriguées pour chacun des sept groupes de parcelles, on obtient des résultats tout aussi précis. Le premier casal, d'une surface totale de $63848 \mathrm{~m}^{2}$ reçoit $\mathrm{I}_{45} \mathrm{O}, 5 \mathrm{O}$ minutes $(24 \mathrm{~h} \mathrm{I} 7)$; le deuxième, de 65402

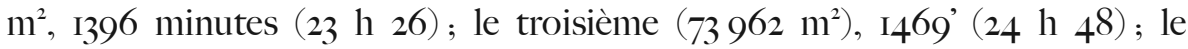

\footnotetext{
7 Vázquez Martínez note que les sept jours de la semaine et ses nuits ne représentent que 168 heures, expliquant que la différence de $2 \mathrm{~h} 22$ ' provient des parcours considérés comme "restes " d'eau, lors des écoulements dans les rigoles secondaires, lesquels sont aussi décomptés (VázquezMartínez 1946 : 179).
} 
quatrième $\left(69863 \mathrm{~m}^{2}\right), \mathrm{I} 393^{\prime}$ ( $23 \mathrm{~h} \mathrm{2I}$ ); le cinquième $\left(8873^{8} \mathrm{~m}^{2}\right), \mathrm{I}_{53} \mathrm{8}^{\prime}(26 \mathrm{~h}$ $03)$; le sixième $\left(76545 \mathrm{~m}^{2}\right), \mathrm{I} 440,5 \mathrm{O}^{\prime}(24 \mathrm{~h})$; et le dernier (IO7 7I4 m²), I527' ( 25 h 45). Sur ce dernier cadastre, le plus grand propriétaire d'eau est devenu la municipalité d'Arbo, avec 3II, 5 minutes ( 5 h 19), sans doute à la suite d'une redistribution de l'eau et des parcelles lors de la cimentation de la rigole; le plus petit temps d'eau reste de 0,5 minute.

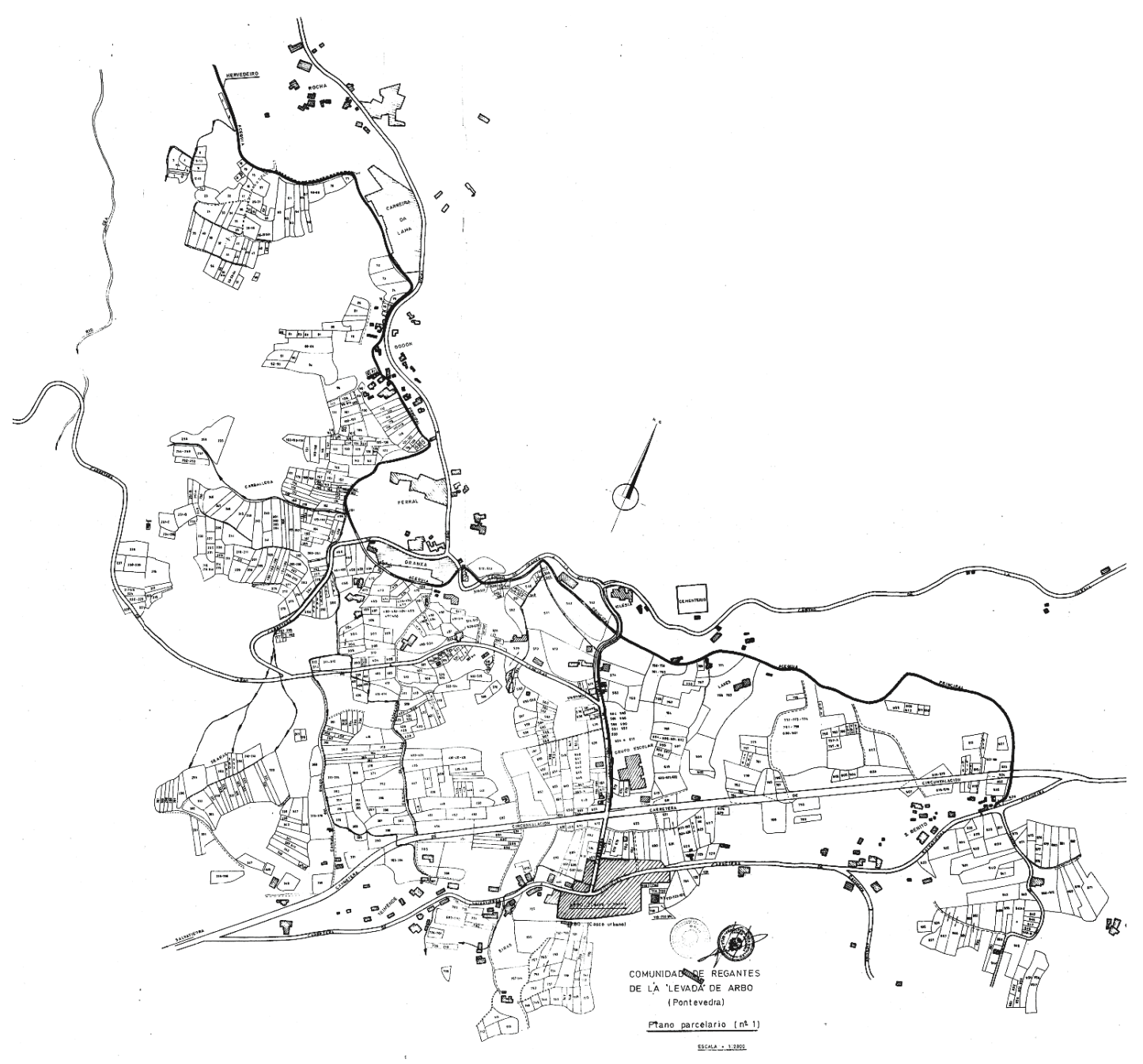

Figure 3. Plan du parcellaire irrigué de la rigole d'Arbo, avec ses sept groupes (casales)

(C) Freijanes Morales, 1988). 
Cette comptabilité extrêmement minutieuse des temps d'eau, associée à une codification sonore précise, a été maintenue durant cent cinquante ans au moins, en admettant des variations minimes, mais aussi et surtout en entretenant une mathématique complexe qui semble comme avoir été protégée au fil des ans. L'existence d'une telle précision semble vouloir renforcer limportance de cette activité estivale au sein de la communauté, comme celle de sa nécessaire organisation et de son impact ou effet sur l'organisation sociale à Arbo. Mais quelle place la conque aujourd'hui délaissée a t-elle occupée au sein de cette organisation ? Quelles informations nouvelles l'étude de cet instrument d'appel en particulier peut-elle nous donner sur la société ? Face à l'absence d’informations directes, revenons donc à des considérations plus générales sur les conques pour tenter d'envisager les pistes interprétatives possibles et étayer quelques hypothèses.

\begin{tabular}{|c|c|c|c|c|c|c|c|c|}
\hline & Casal 1 & 2 & 3 & 4 & 5 & 6 & 7 & Total \\
\hline $\begin{array}{c}1946 \\
(1843)\end{array}$ & $24 \mathrm{~h} 20$ & $23 \mathrm{~h} 32$ & $23 \mathrm{~h} 58$ & $24 \mathrm{~h} 22$ & $24 \mathrm{~h} 04$ & $23 \mathrm{~h} 32$ & $26 \mathrm{~h} 17$ & $170 \mathrm{~h} 22$ \\
\hline $\begin{array}{c}1975 \\
(1952)\end{array}$ & $24 \mathrm{~h} 17$ & $23 \mathrm{~h} 29$ & $24 \mathrm{~h} 52$ & $23 \mathrm{~h} 30$ & $26 \mathrm{~h} 28$ & $24 \mathrm{~h} 10$ & $26 \mathrm{~h} 00$ & $171 \mathrm{~h} 28$ \\
\hline 1988 & $24 \mathrm{~h} 17$ & $23 \mathrm{~h} 26$ & $24 \mathrm{~h} 48$ & $23 \mathrm{~h} 21$ & $26 \mathrm{~h} 03$ & $24 \mathrm{~h} 00$ & $25 \mathrm{~h} 45$ & $170 \mathrm{~h} 23$ \\
\hline
\end{tabular}

Figure 4. Tableau récapitulatif des temps d'eau distribués par la rigole d'Arbo, par dates de cadastres et groupes de parcelles ${ }^{8}$.

\footnotetext{
8 On pourrait entrer davantage dans le détail de cette comptabilité si l'objet de cet article était l'irrigation et non pas la conque en particulier. S'agit-il d'une anomalie ou d'une erreur dans le cadastre de 1975, corrigé à la main? Une heure de différence dans les totaux (en 1975) représente une quantité d'eau assez élevée. Une étude plus approfondie aurait pu fournir des éléments de réponses et des informations plus précises sur l'irrigation et notamment sur le nombre et les types d'usagers de l'eau, les écarts dans la possession du foncier, les disparités par rapport à la taille moyenne des parcelles (ici de $649 \mathrm{~m}^{2}$ ) et, conséquemment, des temps d'eau, etc. Je remercie José Simón, ancien responsable de la Confédération hydraulique à Arbo, de m'avoir permis l'accès et la photocopie des différents cadastres.
} 


\section{Instrument de musique, objet sacré}

Dans la littérature anthropologique où il est question de conques, ou même au sein des collections recensées dans les musées, ces objets sont le plus souvent considérés comme des instruments de musique ou des instruments de signal ou d'appel. Leur aire de répartition est très vaste, s'étendant de l'Océanie à l'Amérique en passant par l'Inde, le Tibet, le Japon, l'Indochine, la Nouvelle-Guinée, la Nouvelle-Zélande, l'Europe. Leur ancienneté est aussi avérée, certains modèles composés étant antérieurs, en Inde, au $\mathrm{II}^{\mathrm{e}}$ siècle (Rouget $1948: 298$ ). Les instruments de musique sont sans doute les plus représentés en nombre, ou pour le moins les plus visibles, pour avoir retenu plus facilement l'attention des voyageurs et des chercheurs. Simplement percées d'un orifice ou composée d’un embout (un «tuyau insufflateur») de matériau pouvant varier selon les lieux et les destinations9, les conques se présentent, d'un point de vue organologique, sous deux formes. On les appelle «terminales » quand l'embouchure a été obtenue en sectionnant la pointe de la coquille et «traversières " quand l'embouchure est un trou percé sur le côté du coquillage (Ibid. : 297). Ces conques sont des aérophones, des instruments à vent généralement fabriqués dans un gros coquillage de mer, telle la fameuse antsiva de Madagascar pouvant peser jusqu’à 900 grammes, ou les conques des ensembles Tongan de Polynésie qui servaient à accompagner le déroulement des matchs de cricket (McLean I999:362).

Mais les conques auraient été choisies, selon Gilbert Rouget, "moins pour leurs qualités acoustiques que comme objets auxquels se rattachent certaines représentations magiques ou religieuses" (Rouget 1948 : 298). Les conques possèdent une fonction essentiellement rituelle, avance-t-il, surtout parmi les plus rares d'entre elles, celles à l'enroulement de la coquille se faisant sur la gauche, dans le sens contraire des aiguilles d'une montre. Des conques sénestres en poterie et en bronze ont été fabriquées et retrouvées en Amérique et en Asie, « sans doute pour leur conférer un plus grand pouvoir rituel » (Ibid. : 299). Ces objets sont la propriété exclusive des prêtres pour jouer un rôle important et précis lors d'une cérémonie ou simplement pour

\footnotetext{
${ }^{9}$ Selon le corpus étudié par Gilbert Rouget : de métal au Tibet, Japon, Indochine et Amérique ; de bambou en Inde, Nouvelle-Guinée, Mexique; ou de cire chez les Tcham de l'Indochine et les Indiens Ijca et Chibcha (Rouget $1948: 299$ et 304).
} 
appartenir au registre du sacré. Les célèbres conques d'Unkar invitaient les moines tibétains à se rendre au temple au lever du soleil; le personnage mythique Triton, connu pour être le trompette de son père Poséidon, annonçait ce dernier en soufflant dans une conque, laquelle lui servait aussi à calmer les flots, apaiser les tempêtes, retenir les eaux du déluge ou encore effrayer les géants. La conque, symbole de pouvoir, est aussi l'attribut de Vishnu, de Shiva et de Bouddha, qui représentent le dieu créateur. Par association, elle est liée au roi, qui sort ou entre dans les villes « au son des conques mêlé au bruit des tambours" (Marcel-Dubois I94I : IOI); elle est « un symbole de la royauté à Samoa [...], la voix de l'autorité ${ }^{-}$son nom en Mélanésien, pu ou pu u’á, recouvrant même la signification de "chef" (McLean I999: 155, 362 et 62). Dans toute l'Océanie, la Polynésie et la Mélanésie, elle est également un instrument belliqueux fort effrayant: " longtemps la conque servit dans les combats pour ramener auprès des chefs les guerriers dispersés (Ibid. : IOI). Et selon le navigateur Cook : «le son des conques que nous avions entendu le matin, était un signal de défi " (Cook cité par Schaeffner I968: 258). Elle joue aussi un rôle important dans les rites de fertilité, résonne pour le mariage de la fille aînée du roi, sert pour les rituels de mort et d'initiation des garçons, ou encore lors de la fête de la première grossesse (Rouget 1948 : 300-30I); elle salue la remise de cadeaux (Malinovski cité par Schaeffner 1968: 258). Les voyageurs et premiers ethnographes, tels que Cook, Malinowski, Leenhardt ou Mauss, ont largement commenté l'usage symbolique et magique de ces grandes conques insulaires, dont certains exemplaires aujourd'hui sont retrouvés dans les collections ethnographiques des musées. Au musée du quai Branly, à titre indicatif, le détail des fiches donne encore ces autres utilisations ${ }^{10}$ : l'ornement et la protection des maisons mélanésiennes; l'ornement et la protection des corps (pendentif, bracelet, boucle d'oreille, collier, cache-sexe, etc.) ; la décoration des statuettes de divinités ; l'efficacité des récipients votifs en forme de conque ou comportant des dessins de conques sur leurs faces.

La petite conque d'Arbo, terminale, simple, sans tuyau insufflateur, ne ressemble guère à un instrument de musique. Pas plus qu'elle ne semble associée à une fonction ou à une vertu magico-religieuse particulière. Elle est dextre et non pas sénestre; elle n'appartient ni à un prêtre ni à un chef; elle

10 Je remercie Sarah Frioux-Salgas pour son aide à la consultation des données du catalogue de la documentation muséale. 
ne sert ni pour la guerre ni pour des rites de fertilité. Par ailleurs, aucun discours de nature symbolique ne m’a jamais été rapporté à son propos. Rien ne nous permet done de dire quil s'agit d'un instrument à connotation symbolique. On pourrait bien sûr imaginer que la conque appelle l'eau qui fertilise la terre et que le choix de cet instrument renvoie directement à la corne d'abondance tenue par Ploutos, le dieu grec de la richesse et de l'abondance. Mais cela ne serait que pure et stérile spéculation. Tout invite plutôt à confirmer lidée que la petite conque d'Arbo est un objet profane et domestique, tout comme les deux autres instruments utilisés aussi en période d'irrigation. La corne est avant tout un instrument d'appel.

\section{Objet profane, instrument d'appel}

Les usages qui renvoient aux activités de nature plus profane sont aussi largement rapportés dans la littérature. La conque, à linstar des cornes de bovins ou de caprins, est un instrument de signal et d'appel fort courant et répandu. Elle est d’ailleurs utilisée par différents corps de métier. En bord de mer, elle servait aux pêcheurs et aux marins : la conque annonçait le départ et l'arrivée des marins à Madagascar ${ }^{11}$; sur le littoral portugais, il est précisé qu'elle annonçait les pêches fructueuses et servait aussi de corne de brune ${ }^{12}$; aux Açores, elle signalait la présence des baleines ${ }^{13}$. Dans le port de Bastia, en I87I, « les conducteurs de trains de remblais du petit chemin de fer servant à l'entreprise du port n'avaient point d'autre instrument pour prévenir de leur passage" que la conque (Locard cité par Schaeffner 1968: 17). Dans les terres, en Provence, les ouvriers l'employaient pour avertir alentour quand on faisait partir les mines (Armengaud I984: 72). Au Portugal, dans l'intérieur sec et aride, c'est le fromager qui en sonnait pour annoncer le petit lait prêt à consommer (fig. 5) ; tandis qu’en Polynésie, pour annoncer sa fournée, c'est le boulanger qui en usait (McLean 1999: 62). Aux Açores, le meunier en sonnait pour signaler qu'il n'y avait plus de grain à moudre. En Crête, elle est retrouvée entre les mains des gardes-champêtres (Glotz cité par Schaeffner I968 : I7) ; à Madagascar, entre celles des pompiers qui signalent l'incendie.

\footnotetext{
${ }^{11}$ Voir les archives en ligne du musée d'Art et d'Archéologie de l'université d'Antananarivo, Madagascar

12 Voir une conque à appeler les pêcheurs dans les vitrines du Musée municipal de Santiago de Cacém, Portugal.

${ }^{13}$ Conque des réserves du musée national d'Ethnologie de Lisbonne, Portugal.
} 


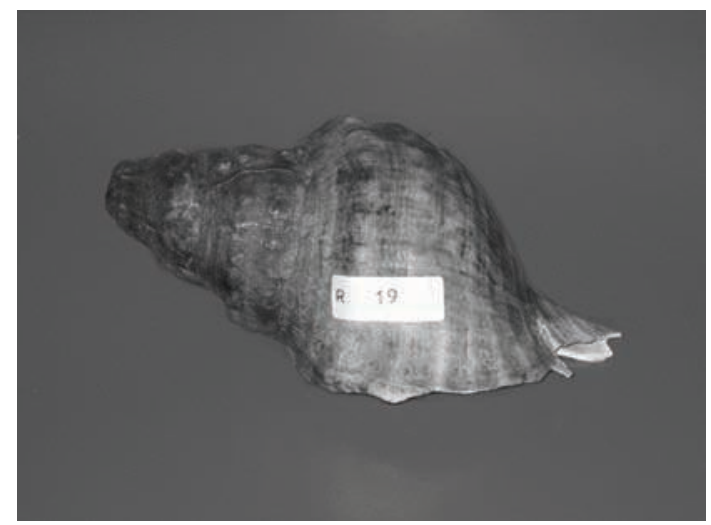

Figure 5. Conque pour appeler au petit lait. Musée ethnographique de Serpa, Portugal (cliché Fabienne Wateau).

Dans le monde pastoral et agricole, les destinations sont encore variées. Vers la fin du XIXe siècle, les bergers corses et sardes se seraient servis de conques à embouchure terminale pour communiquer entre eux (Ibid. : I7). Les fiches de l'ancien musée des Arts et Traditions populaires à Paris parlent aussi de conques utilisées pour rassembler le bétail en Haute-Corse et en Camargue, ou pour réveiller les gens pour la traite des brebis à quatre heures du matin dans le Tarn-et-Garonne ${ }^{14}$. Celles du musée national d'Ethnologie à Lisbonne mentionnent lappel pour la réunion des membres de la communauté à l'occasion des travaux collectifs (Lopes de Oliveira I97I : I64), comme la cueillette des olives dans la région Alentejo, ou encore chaque matin au lever du soleil, pour assurer la garde du bétail par roulement ${ }^{15}$. Enfin, des conques existent pour la pratique de lirrigation, bien qu'un seul autre exemple ait été retrouvé dans la littérature anthropologique, dans l'ouvrage d'Olivia Aubriot sur le Népal : «à cette époque, toute l'eau est mise dans un seul canal à la fois, pendant 24 heures d'affilée et, à un instant donné, cette eau est utilisée par un seul riziculteur. La fin de la durée d’irrigation est

\footnotetext{
14 Je remercie Marie Janet-Robert, conservateur, pour l'accès aux fiches du musée des Arts et Traditions populaires aujourd'hui fermé et dans l'attente de sa réouverture à Marseille en musée des Civilisations de l'Europe et de la Méditerranée.

15 Je remercie Joaquim Pais de Brito pour l'accès aux archives et à la documentation sur les huit conques des réserves du musée national d'Ethnologie ainsi que pour la relecture de ce texte.
} 
annoncée par le son émis par une conque, utilisée comme trompe » (Aubriot 2003 : 20I).

Observons que l'on utilisait indifféremment la conque ou la corne de bœuf pour sonner, précise Jorge Dias, «l'usage de la trompette de corne, ou de bois, vient des temps néolithiques; il a dû être répandu en Europe, par l'intérieur de l'Asie, par les peuples de culture de bétail " (Dias I948 : 89). Retenons aussi que c'est le son puissant de la corne ou de la conque qui compte ici, voire le vacarme qu'elles peuvent faire. La conque, objet domestique profane, sert aussi et parfois, à des moments très précis du calendrier festif, d'instrument sonore pour effrayer et pour éloigner. En Corse, c'est l'instrument des ténèbres et du vacarme de la période de carnaval et de carême, destiné à remplacer les cloches alors interdites durant ces périodes (Verdoni 2003: 232). Au Portugal, c'est linstrument qui accompagne les personnages carnavalesques de Vale de Ilhavo lors du sermon burlesque, ou encore lors de linterruption du carême ${ }^{16}$. La conque népalaise pour l'irrigation servait aussi aux brahmanes lors de rituels et de l'annonce des décès (Aubriot 2003:201).

La petite conque d'Arbo est incontestablement un objet profane, domestique, qui servait à signaler les tours d'eau d'irrigation. Aurait-elle pu être remplacée par une corne animale ? Oui sans doute. Car la combinaison de conque et de corne animale utilisées à de même fins d'appel et de signalisation a déjà été confirmée. C'est le cas à Vilarinho da Furna, par exemple (fig. 6). Néanmoins, aucune corne animale n’a été retrouvée à Arbo, et personne n'a mentionné cette éventuelle association entre la conque et la corne. La petite conque d'Arbo a-t-elle déjà servi en période de carnaval comme instrument de vacarme ? Peut-être. Mais à l'état d'avancement de cette recherche, rien ne permet de l'avancer ici.

${ }^{16}$ Dans la région d'Aveiro, Portugal, d'après les fiches du musée national d'Ethnologie. 


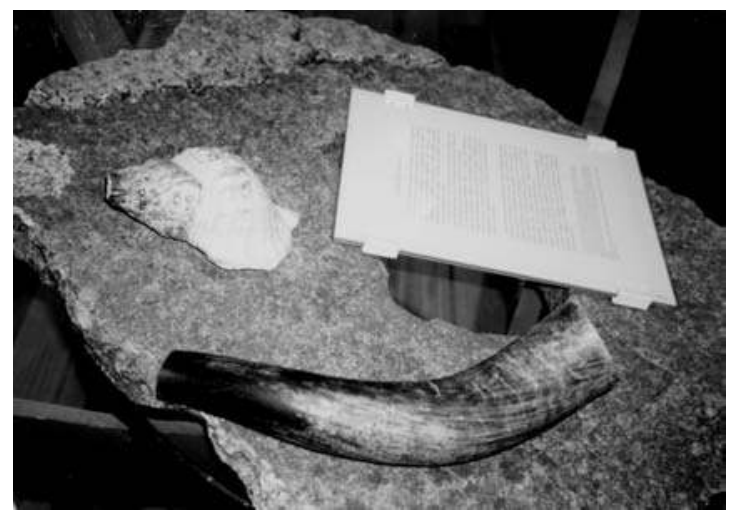

Figure 6. Conque et corne de bœuf qui appellent aux travaux collectifs, exposées ensemble sur une meule de granit. Musée de Vilarinho da Furna, Portugal (cliché Fabienne Wateau).

\section{Souffler pour ordonner}

Revenons maintenant au profil du sonneur pour tenter de mieux comprendre ce que la conque peut donner à voir de la société. Le levador d'Arbo, on l'a vu, n'était pas un simple souffleur de conque. Responsable de la rigole, c'est à lui qüincombait le bon déroulement de lirrigation en été, avec la meilleure efficience possible, en évitant le maximum de conflits. Sa tâche n’était done pas forcément aisée. Pour autant, sa fonction ne renvoyait pas véritablement à un statut privilégié conférant du prestige et une autorité. Certes, "tous les ayants droit devaient respecter le levador et se considérer sous ses ordres, dans le cadre de ce qui avait été traditionnellement établi. Les deux levadores avaient une autorité pour élucider, résoudre, éclairer et devenir des témoins privilégiés, en cas de conflits graves, quand la justice municipale devait intervenir, ou quand une résolution à l'amiable n'avait pu être trouvée " (Vázquez Martínez 1946: 174). Mais dans la réalité et dans la mesure du possible, le levador évitait d'avoir à exercer son autorité. La sanction contre un irrigant était extrêmement rare. C'est pour le moins ce que m’a expliqué Pépé qui ne présentait absolument pas le profil d'une personne autoritaire, mais plutôt celui d'un villageois modeste à la fois compétent et disponible pour s'occuper de la rigole tout l'été. Célibataire, comme certains autres sonneurs que jai pu rencontrer par la suite, sa parfaite connaissance du parcellaire irrigué et son plus grand temps libre faisait de lui un levador idéal. 
En cas de conflits, c'est à l'ensemble de la communauté que l'on renvoyait, et parmi les personnages plus importants encore que le levador se trouvait le président de l'association des irrigants pour délibérer. Le levador n'est done pas un chef de lirrigation; la conque n'est pas un attribut de pouvoir ou de prestige. Néanmoins, le détenteur officiel de la conque avait pour interlocuteur régulier chacun des irrigants de la rigole, qu’il connaissait parfaitement bien. Envers lui, des relations de nature ordonnée, pour le moins, pour le bon fonctionnement de la distribution de l'eau, et hiérarchisée peut-être, devaient sétablir. Potentiellement, le personnage était en mesure d'installer une hiérarchie même provisoire au sein de la communauté. Pour autant, sa conque n'en devient pas un instrument de pouvoir ou de prestige.

Ce que nous donne à voir l'existence et l'emploi de cet instrument, c'est une organisation sociale extrêmement dirigée et centralisée. Et cela est d'autant plus flagrant qu'une comparaison avec le village voisin de Chaviães, côté portugais, est possible. En Espagne, à Arbo, le système de distribution de la rigole a été pensé de façon à être le plus efficace et économe possible. L'eau d'irrigation captée directement au pied des sources ou détournée depuis la rivière est depuis fort longtemps systématiquement acheminée jusqu'à la rigole principale par une canalisation cimentée ou un tuyau étanche de plastique. La déperdition est évitée au maximum. Quand ils sont naturels, les chemins de l'eau sont aussi protégés : on facilite ainsi la traversée d'une route en ajoutant des gouttières; on installe régulièrement sur les tracés des bassins-réservoirs cimentés qui retiennent les eaux excédentaires. La planche du parcellaire irrigué indique quant à elle et avec précision les parcelles bénéficiaires, en leur attribuant un numéro qui s'avère être aussi un numéro d'ordre de passage dans la distribution de l'eau. Les parts d'eau distribuées sur les parcelles sont équitables et proportionnelles à la taille des terres. Les écrits, enfin, sont nombreux et respectés : tous les droits d'eau sont écrits et recensés dans les archives de l'association des irrigants. Ces droits écrits font office de preuve et de documents en cas de litige. Pour terminer, un responsable de l'irrigation sillonne jour et nuit le parcellaire irrigué en période d'irrigation. Sa fonction de levador l'invite à surveiller l'ordre des tours d'eau, soit à ordonner l'irrigation et avec elle la société, au moins le temps d'un été. En d'autres termes, en Espagne, il y a bien longtemps que les ingénieurs agricoles agissent sur le foncier. Ils sont reconnus et valorisés. Sans doute l'explication est-elle en partie politique, car l'Espagne applique 
une politique hydraulique forte depuis plusieurs siècles déjà, en en faisant même dès le XIX ${ }^{\mathrm{e}}$ siècle avec Joaquim Costa puis sous la dictature avec Franco, un de ses axes forts de développement par l'irrigation de la société espagnole.

Tel n'est pas forcément le cas au Portugal où le manque d'eau à l'échelle nationale est comparativement moindre et où les ingénieurs agricoles sont, encore et le plus souvent fort mal reçus et renvoyés à leurs logiques strictement économiques. Côté portugais, en effet, pour un parcellaire irrigué de taille comparable, sur la commune de Chaviães irriguée par la rigole de la Candosa, les logiques et les principes qui président à la distribution de l'eau ne sont pas ceux de l'optimisation économique de la ressource. Là, le partage de l'eau se fait en fonction d'une logique sociale et notamment d'une histoire de l'occupation des sols et d'une représentation hiérarchisée des espaces : très concrètement, est irrigué en premier celui qui est arrivé le premier. Les terres situées près de l'église ou de la route romaine, premiers foyers d'occupation humaine, reçoivent toujours en premier l'eau de la rigole, et ce, qu'elles que soient leur localisation par rapport à l'amont et à une logique gravitaire. La déperdition de l'eau, avec ce système social, est certaine. Mais cette logique continue d'être appliquée et est vivement revendiquée. De même, la planche du cadastre indique-t-elle des numéros de parcelles mais pas un ordre de passage. Les parts d'eau ne sont pas non plus proportionnelles à la superficie des parcelles, car ici «l'eau et la terre sont célibataires ", c'est-à-dire que leurs droits sont distincts et qu'une terre peut alors bénéficier de l'eau originellement destinée à une autre parcelle, au bon vouloir de son propriétaire, créant ainsi des parts d'eau des plus insolites. Enfin les écrits ne font pas foi. En général, il n’existe que très peu d'écrits sur lïrrigation au Portugal, "l'eau ne s'écrit pas" précisait-on à Melgaço. Et quand ils existent, comme cette sentence établie par les services de l'hydraulique de Porto pour tenter de résoudre des conflits sur le trajet de la Candosa, ils ne sont pas pris en compte, leur pertinence n'est pas reconnue. L'oral occupe une place plus importante que l'écrit dans cette société de type agonistique, sans doute pour lui laisser l'occasion de pouvoir s'exprimer par le conflit ${ }^{17}$.

\footnotetext{
17 Sans entrer ici davantage dans les détails, je renvoie à mon ouvrage sur l'irrigation et les conflits à Melgaço
} (Wateau 2002). 
La question renversée que je me pose alors est la suivante : pourquoi côté portugais les irrigants n’ont-ils pas adopté la conque ? Étant entendu qu’ils ont partagé, à l'instar de leurs voisins, les usages de la pierre de partage et de la canne à mesurer l'eau. Est-ce parce que leurs organisations sociales -et en particulier leurs logiques- sont tellement différentes qu'elle ne permettent pas d'adopter certaines des options choisies par leurs voisins? Là se trouve une explication possible. Il existe aussi des levadores côté portugais (le terme est exactement le même en galicien et en portugais), censés s'occuper du bon fonctionnement de la rigole cimentée de la Candosa, et notamment d'en vérifier l'état. Mais ces levadores, tout d'abord, ne sont pas en contact avec l'ensemble des irrigants. Ensuite, ils ne sont pas des responsables de la rigole mais plutôt des techniciens du réseau. Ce qui signifie aussi qu'en cas de conflits, ils laissent les gens se débrouiller entre eux; jamais lun d'eux ne se charge ou n'est chargé d'indiquer le roulement des tours d'eau. À Chaviães, comme dans toute la vallée de Melgaço, chaque irrigant fait exactement tout ce que font les autres. Le savoir et les savoir-faire sont partagés; et aucune personne en particulier n'est déléguée à une fonction particulière d'ordre collectif. Par crainte de linstauration possible de hiérarchies nouvelles, précisément, les places de chef ou de responsables ne sont ni souhaitées, ni véritablement recherchées. On sait reconnaitre des savoirs et des compétences à un autre que soi, mais cette personne n'en deviendra pas pour autant plus responsable du groupe qu’un autre. Aussi le système régulé à l'aide d'une conque, placé entre les mains d'une même personne, ne semble-til pas convenir aux habitudes et logiques melgacenses. Car la conque pourrait bien apparaitre ici comme un attribut trop distinctif, un instrument de pouvoir. Et en cela elle se détache très nettement des pierres et des cannes, où les savoirs et leurs applications sont toujours partagés à l'occasion du regroupement collectif de tous les ayants droit. L'hypothèse peut paraître fragile et difficilement vérifiable, pourtant, elle pourrait bien faire sens au regard des travaux cognitifs déjà déployés dans cette vallée.

\section{Sonoriser l'espace rural}

Pour finir, jaimerais revenir sur la dimension sonore et créatrice de lien social de notre objet. Quand je suis retournée sur le terrain en 2003, avec lintention de filmer la conque dans son contexte géographique -faute d'un contexte 
social et d'une activité agricole encore existants- il était difficile de reconnaitre les anciennes terrasses dans le paysage ou simplement d’imaginer, quil y a vingt ans à peine, toutes les terres étaient intensément cultivées et le parcellaire de la rigole d'Arbo parfaitement délimité. Aux premiers sons de conque, pourtant, tous se souvenaient avec une certaine nostalgie de cette sonorité particulière et saisonnière, de cette pratique estivale de liirrigation qui réunissait les irrigants et les invitait à rester attentifs aux signaux que leur lançait le levador. Ce son centripète qui unifiait et ordonnait la communauté - à l'instar des cloches des horloges ${ }^{18}$ ou du bruit familier du moulin, centre de la vie du bourg, dont a pu parlé Murray Schafer (I979: 88), ou encore du grincement caractéristique des anciens chariots en bois de tractions animales, aux timbres parfaitement distingués et de surcroît identifiés à leurs propriétaires respectifs ${ }^{19}$-, créait un véritable lien social entre les villageois. Pas seulement entre les bénéficiaires d'eau de la rigole d'Arbo, mais entre tous les villageois, et sans doute avec eux ceux du village voisin de Barcela, si l'on en croit leurs propos, qui disent aussi se souvenir de ce souffle entendu au loin dans la vallée du Cea. Le son de la conque pouvait s'entendre jusqu'à cinq kilomètres affirme le dernier responsable de la rigole d'irrigation, par temps clair, glissant dans la vallée sans retenue, dans une réverbération longue et en déplacement qui propageait ses décibels jusqu'aux oreilles les plus éloignées. Tandis que l'œil s’adresse à l'homme extérieur, l'oreille attire au-dedans; elle absorbe linformation, elle s'adresse à l'homme intérieur expliquait Schafer (1979:26). Et plus qu'un signal ou une figure que l'on écoute consciemment, attentif, le son de la conque était devenu une empreinte sonore de ce paysage rural, ce son unique à la sonorité précise et partagée qu’avaient pris en considération et intégré les membres de la communauté. Cette sonorité qui permettait aussi de souligner une occupation sociale et sonore d'un groupe dans l'espace, d'en donner ainsi une certaine identification et représentation. Les saisonniers agricoles du sud du Portugal lutilisaient comme moyen de communication, pour prolonger leur présence et pour étendre et maintenir le plus longtemps possible, grâce au son, le lien communautaire qui les reliait eux, migrants temporaires, à leur familles et voisins restés au village. Par le

\footnotetext{
18 Les cloches des églises étaient arrêtées pendant la période d'irrigation, rapporte la littérature ibérique. Voir l'argumentation développée à propos de l'horloge solaire ou pierre de partage de l'eau dans Wateau 2006a.

19 Joaquim Pais de Brito, observations à Rio de Onor (P) dans les années 1980, communication personnelle.
} 
son, on savait aussi quills étaient bien arrivés à destination; par le son, leurs hôtes pouvaient s'apprêter à les accueillir: "Les groupes de moissonneurs qui se rendaient dans la région Alentejo soufflaient dans la conque durant toute leur traversée de la montagne. Cesser d'entendre la conque au village signifiait que les saisonniers étaient bien entrés dans les terres de l'Alentejo » (Prista I993 : 126).

Pour autant, la conque reste un objet banal. Elle sert à tout, comme on a pu le voir, aux quatre coins du monde. De petite ou de grande taille, à connotation magique ou simplement profane, c'est sans doute et néanmoins sa sonorité singulière, à la fois profonde, puissante et à réverbération longue, qui fait que nombre de sociétés l'ont choisie et lui ont conféré un intérêt particulier. Que l'on se trouve en bord de mer ou à lintérieur des terres, en plaine comme en montagne, ses destinations sans limitation de possibilités sont difficilement quantifiables. Seul son éclat semble avoir été unique. Ici la conque servait à ordonner lirrigation estivale. Cet exemple parmi d'autres aura permis de rappeler limportance des fonctions fédératrices et organisationnelles de cet instrument sonore, simple de facture, mais à la portée sociale sans doute beaucoup plus vaste encore que ce que la littérature anthropologique - et pas seulement ethnomusicologique- ne lui a jusquà présent accordée. Sans doute faudra-t-il y revenir.

F. W.

\section{Références}

Armengaud, Christine

1984 Musiques vertes. Le Puy : Christine Bonneton Éditeur.

Aubriot, Olivia

2004 L'Eau, miroir d’une société. Irrigation paysanne au Népal central. Paris : Éditions du CNRS.

Dias, Jorge

I948 Vilarinho de Furna. Uma aldeia comunitária. Lisboa : Casa da Moeda - Imprensa nacional.

Freijanes Morales, Jorge-Valentin

I988 Comunidad de regantes de la levada de Arbo (Pontevedra). Colégio oficial de ingenieros tecnicos y peritos agricolas, Pontevedra, Vigo (non publié). 
Lopes de Oliveira, António

I97I Terras de Bouro e o seu concelho. Terras de Bouro : Ed. Câmara municipal de Terras de Bouro.

McLean, Mervyn

1999 Weavers of Song. Polynesian Music \& Dance. Auckland : Auckland University Press.

Prista, Pedro

1993 Sítios de Querença. Morfologias e processos sociais no Alto barrocal algarvio. Thèse de doctorat non publiée, Lisboa, Instituto Superior de Ciências do Trabalho e da Empresa.

Rouget, Gilbert

I948 "La conque comme signe des migrations océaniennes en Amérique ", in Actes du XXVIII congrès international des américanistes. Paris : musée de l'Homme : 297-305.

Shaeffner, André

I968 Origine des instruments de musique. Introduction ethnologique à lhistoire de la musique instrumentale. Paris : Maison des sciences de l'homme/La Hayes : Mouton : 257-26I.

Schafer, R. Murray

1979 Le Paysage sonore. Paris : J.-C. Lattès.

Vázquez Mártinez, Alfonso

I946 "Las levadas de Arbo », Rusco de Pontevedra, 4 : I72-I85.

Verdoni, Dumenica

2003 A Settima Santa in Corsica. Une manifestation de la religiosité populaire. Gap : Albiana.

Wateau, Fabienne

$200 \mathrm{I}$ «Objet et Ordre Social. D'une canne de roseau à mesurer l'eau aux principes de fonctionnement d'une société rurale », Terrain 37 : I53-I6I.

2006 a "La pierre de partage de l'eau. Apprentissage, logique, mesure ", Annales de la Fyssen, 21 : 75-89.

\section{Références filmographiques}

Wateau, Fabienne

2004 Les Conques d'Arbo. 7. Cellule audiovisuelle CETSAH/CNRS, Meudon, CNRS Images.

2006b La Canne à mesurer l'eau. 28'. Cellule audiovisuelle CETSAH/CNRS, Meudon, CNRS Images.

Wateau, Fabienne \& Ana Margarida Campos

2002 La Pierre de partage de l'eau. Io'. Paris : Éditions du Laboratoire d'ethnologie et de sociologie comparative de Nanterre/Lisbonne : musée national d'Ethnologie de Lisbonne. 